\title{
AOR
}

Selected Papers of \#AolR2020:

The 22nd Annual Conference of the

Association of Internet Researchers

Virtual Event / 13-16 Oct 2021

\section{SAFE FROM "HARM": THE GOVERNANCE OF VIOLENCE BY PLATFORMS}

\author{
Julia R. DeCook \\ Loyola University Chicago \\ Kelley Cotter \\ Pennsylvania State University \\ Shaheen Kanthawala \\ University of Alabama
}

\section{Introduction}

Since the early days of the COVID-19 pandemic, and more recently, the January 6 U.S. Capitol Insurrection, a number of issues have emerged in regard to how platforms moderate and mitigate "harm" on their services. Although in recent years platforms have developed more explicit policies in regard to what constitutes "hate speech" and "harmful content," the unintended consequences and side effects about how platforms define harm and how those definitions, in turn, affect users' normative understandings of harm are understudied. Namely, it appears that platforms often use subjective judgments of harm that specifically pertains to spectacular, physical violence - but harm takes on many shapes and complex forms. The politics of defining "harm," "violence," and "danger" within these platforms are complex and dynamic and represent entrenched histories of how control over these definitions extend to people's perceptions of them (Arendt, 1970; Bourdieu, 1999).

With this analysis, we suggest that platforms' narrow definitions of harm, violence, and danger are not just insufficient, but result in these platforms engaging in ideological hegemony, imposing conceptions of not just what violence is and how it manifests, but who it impacts and by what mechanisms. Through this governance, they continue to control normative notions of harm and violence, effectively managing perceptions of their actions (Gillespie, 2017) and directing users' understanding of what is "harmful" versus what is not. Rather than changing the mechanisms of their design that enable harm, the platforms reconfigure intentionality and causality to try to stop users from being "harmful," which, ironically, perpetuates harm.

\section{Data Collection and Method}

We collected data in the form of public facing documentation (such as blog, policy, and help page documents) put forth by three of the major platforms (Twitter, Facebook, and YouTube)

Suggested Citation (APA): DeCook, J. R.. Cotter, K., Kanthawala, S. (2021, October). Safe from "Harm": The Governance of Violence by Platforms. Paper presented at AoIR 2021: The 22nd Annual Conference of the Association of Internet Researchers. Virtual Event: AolR. Retrieved from http://spir.aoir.org. 
that mentioned "harm" and its variants (e.g. violence, hate, etc.). Through keyword searches we assembled a corpus of 270 documents across all three platforms.

Using Bourdieu's symbolic violence framework (1999) and feminist technoscience critiques of "unintended consequences," (Parvin and Pollock, 2020) we conduct a critical discourse analysis of how the three platforms define and police "harm" within their digital milieu. We paid close attention to the power dynamics and asymmetries present (Lazar, 2007), focusing our attention to policy documents about misinformation and hate speech to understand how the platforms are conceptualizing "harm", and their practices to mitigate it via human and machine-driven interventions. Through an iterative process of open coding the documents that mention harm, we tease out not only recurrent or similar practices but the discursive contours of defining and classifying "harmful" content and behavior.

\section{Initial Findings and Implications}

There are three key findings from our initial review of platform policy documents. First, we characterize the platforms" use of the terms "harm" and "violence" as floating signifiers (Mehlman, 1972). Rather than sticking to "fixed" categories constitutive of harm or violence, the platforms seemed to use these terms as concepts that can be molded and interpreted flexibly to fit their needs at any given moment or within a specific context. As new harms emerge and public outcry reaches a tipping point, the platforms adapt their policies accordingly, as in the case of COVID-19 and election misinformation in 2020 (Coppins, 2020; Donovan, 2020). This is apparent in the labels the platforms devise for emergent categories of harm. For example, all three platforms have in recent years begun referring to "coordinated influence operations" (YouTube), "coordinated behavior" (Facebook), and "coordinated inauthentic activity," as catchalls for various harms (e.g., QAnon, election interferences, etc.). While none of the three platforms offered a clear or consistent definition of harm or violence, we did see a patterned emphasis on normative notions of these concepts--namely, an emphasis on child safety, cyberbullying, and terrorism, but less meaningful enagement with other significant forms of harm and violence on their sites.

Second, we observed an inclination to hierarchize and quantify harm and violence, presumably to accommodate the platforms' technical infrastructures. Operationalizing harm and violence in these ways assists automated tracking, identification and moderation of such content, which helps build towards decreased reliance on and investment in human labor. For example, in a blog post, Twitter described a three-tiered system (low, medium, high) to classify the severity of "coordinated harmful activity," which emphasizes the quantity of documentation of such activity. Similarly, Facebook repeatedly refers to "prevalence" as a metric for gauging the magnitude of harm. For example, in a blog post, Facebook explained prevalence, writing: "If a piece of hate speech is seen a million times in 10 minutes, that's far worse than a piece seen 10 times in 30 minutes." Metricizing harm and violence in these ways oversimplifies the complex ways harm manifests and differently impacts different people at different times.

Finally, we saw a discursive positioning of harm and violence as physical and as something that exists outside of the platforms in the "offline" or "real world." In this sense, the platforms seemed to be defining harm and violence narrowly in terms of materiality. Indeed, as mentioned, the platforms consistently referred to child sexual exploitation and/or terrorism as prototypical examples of harmful content. Such an emphasis could be a means of deflecting attention from the less tangible but no less real psychological, emotional, and symbolic violence perpetrated on their sites (Massanari, 2017; Recuero, 2015). For instance, in a report on "harmful stereotypes," Facebook casually stated that the "direct causality" between such content on the 
platforms and "real world" violence is "uncertain." Such rhetoric urges the idea that "real" harm does not occur on the platforms, and, therefore, they should not be held responsible for it.

Our above findings suggest a reactive approach by platforms to defining and addressing harm and violence. By sticking close to normative understandings of harm, they give the appearance of attentiveness while avoiding controversy. Moreover, a positioning of harm and violence in terms of quantifiability and physicality suggests a surface-level approach that underplays the impact and ignores the interrelatedness of different forms of harm and violence (physical, emotional, psychological, symbolic) and of violence and power.

\section{References}

Arendt, H. (1970). On Violence. Houghton Mifflin Harcourt.

Bourdieu, P. (1999). Language and Symbolic Power (J. Thompson, Ed.; G. Raymond \& M. Adamson, Trans.; Reprint edition). Harvard University Press.

Coppins, M. (2020). The Billion-Dollar Disinformation Campaign to Reelect the President. The Atlantic. https://www.theatlantic.com/magazine/archive/2020/03/the-2020-disinformationwar/605530/

Donovan, J. (2020). Social-media companies must flatten the curve of misinformation. Nature. https://doi.org/10.1038/d41586-020-01107-z

Lazar, M. M. (2007). Feminist Critical Discourse Analysis: Articulating a Feminist Discourse Praxis. Critical Discourse Studies, 4(2), 141-164.

Gillespie, T. (2017). Governance of and by platforms. In J. Burgess, T. Poell, \& A. Marwick (Eds.), The SAGE Handbook of Social Media (pp. 254-278).

Massanari, A. (2017). \#Gamergate and The Fappening: How Reddit's algorithm, governance, and culture support toxic technocultures. New Media \& Society, 19(3), 329-346.

Mehlman, J. (1972). The" floating signifier": from Lévi-Strauss to Lacan. Yale French Studies, (48), 10-37.

Parvin, N., \& Pollock, A. (2020). Unintended by Design: On the Political Uses of "Unintended Consequences." Engaging Science, Technology, and Society, 6(0), 320-327.

Recuero, R. (2015). Social Media and Symbolic Violence. Social Media + Society, 1(1), 1-3. 